\title{
Processing of teeth of saw blades of cotton processing machines with a stream of compressed air with abrasive particles
}

\author{
Illarion Shin ${ }^{1 *}$, Zokhir Shodmonqulov ${ }^{1}$, Sakhrobjon Nazarov ${ }^{1}$, and Nigora Iskandarova ${ }^{1}$ \\ ${ }^{1}$ Tashkent Institute of Textile and Light Industry, 100100 Tashkent, Uzbekistan
}

\begin{abstract}
In the article, it is presented materials on abrasive blasting of teeth of saw blades of cotton processing machines with particles of black silicon carbide. The micro-profile of the surface, characterized by the height and pitch of irregularities, after microcutting with abrasive particles is able to intensify the process of fiber separation by activating the tank surfaces of the teeth. This machining process is also accompanied by deformation hardening of the surface layer of the teeth, leading to an increase in the wear resistance and durability of the saws.
\end{abstract}

\section{Introduction}

The main purpose of cotton processing machines is the gin and linter gin separation. While gins remove the bulk of the fibrous material from cotton seeds, linters remove the fibrous cover remaining after the ginning operation from comparatively short fibers, the amount of which depends on the selection and industrial grade of raw cotton. So, for medium-fiber varieties, the amount of fibrous material is $11 \ldots 16 \%$ by weight of seeds, and for fine-fiber varieties - 3...5\% [1-3]. The fibrous material remaining on cotton seeds consists of short fibers ranging in length from $1 \ldots 1.5$ to $25 \ldots 26 \mathrm{~mm}$. In this case, a fiber with a length of 6 $\mathrm{mm}$ or more is referred to as a lint, and a fiber with a length of less than $6 \mathrm{~mm}$ is a delint or short-stapled lint [4].

Technological machines for cotton processing have special requirements for the quality of the surface layer of the working body parts. In gins and linters, the working body is a saw cylinder consisting of discs of the same name $\varnothing 320 \mathrm{~mm}$ in diameter and $0.95 \mathrm{~mm}$ thick, made of $65 \mathrm{G}$ and U8G steels (tensile strength $\sigma=1150 \mathrm{n} / \mathrm{mm}^{2}$, hardness HRA 67-70, elongation $\delta=6 \%$ ) by cold cutting teeth (Ffig. 1) on a special sawing machine $[5,6]$.

The technological preparation of saw blades for work involves a number of preparatory operations: processing in a sand bath, grinding the side surfaces of the teeth, straightening in case of flatness (more than $0.5 \mathrm{~mm}$ ) of the surfaces of the discs. The contact working surfaces of the saw blade teeth entering into force interaction with the raw cotton roller formed in the machine chamber must have a surface roughness of no more than $1,25 \ldots 0,63$ microns [7, 8]. A coarser surface in the process of separating fibers from seeds can dramatically increase mechanical damage to fibers in the form of microscopic cuts or even

* Corresponding author: nigora1211@mail.ru 
to a cut of fibers, which worsens their natural properties (strength decreases, fiber length decreases and, accordingly, the number of short fibers increases), which directly affects the spinning process [9]. The second stage of fiber separation, associated with the removal of the remaining fibrous material, is realized through scraping and cutting of short fibers with a sharp top of the teeth and their edges on the front and side faces, representing transition surfaces. The quality of the teeth and the durability of the saw blades directly affect the efficiency of the ginning machines, which is expressed in the production of high quality products - cotton fibers, linters and seeds. Moreover, saw blades are the most common parts of these machines. So, in linters, their number reaches 160 , and in gins, depending on the design, -90 or 130 [10].

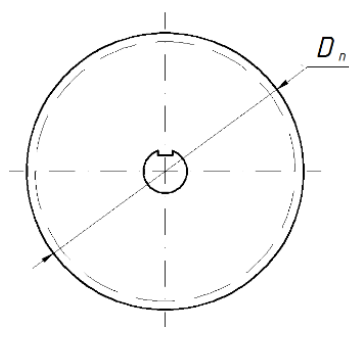

a)

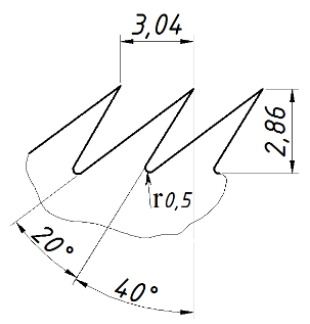

b)

Fig. 1. Linter saw with diameter Ds (a) and profile of teeth with dimensions of geometric parameters (b)

Severe working conditions of saw blade teeth (moisture of raw cotton, presence of weeds in the form of solid abrasive particles - granite, corundum, limestone, high-cycle variable loading) lead to various types of their destruction: abrasive wear, plastic crushing, breakage. These types of destruction reduce the performance of the saw blades, as the teeth lose their main function - gripping power when ginning. Therefore, technological methods of increasing the efficiency of saw blades using strain hardening [11-13] of the surfaces of the teeth seem to be very relevant and necessary for production. Moreover, in accordance with the technical regulations, saw blades must be replaced after 72 and 48 hours of operation, respectively, on gins and linters [14].

If we take into account the value of lint as a raw material and the great demand in various industries (textile, chemical, printing, etc.), then the issue of intensifying the linting process remains important. One of the ways to increase the productivity of linters can be the activation of the side surfaces of the saw teeth by creating on their working surfaces by preliminary machining a corresponding micro-profile with height and step parameters commensurate with the diameters of cotton fiber.

For the purposeful formation of a micro-profile on the lateral surface of the saw blade teeth, capable of actively gripping short fibers and further cutting them off, abrasive blasting was used. Due to its versatility, this type of processing has become widespread in various industries and is carried out on special equipment (abrasive blasting chamber) to solve many technological problems: surface preparation, cleaning, removing rust, scale and surface finishing, deburring, polishing, obtaining the necessary surface roughness, strain hardening (work hardening).

\section{Materials and Methods}

Black silicon carbide (CS) with a grain size of 40 was chosen as an abrasive material. In accordance with the standard content of the main fraction is $45 \%$, which corresponds to the 
range of grain size $200 \ldots 12$ and abrasive grains belong to the abrasive grain. The choice of this abrasive material is due to its high cutting properties and the possibility of creating a favorable micro-profile of the surface as a result of microcutting.

Experimental research were carried out on a special sandblasting machine under the following conditions: compressed air pressure $\rho=0,1 \ldots 0,4 \mathrm{MPa}(1 \ldots 4 \mathrm{~atm})$, attack angle $\alpha=30 \ldots 60^{\circ}$, time of processing $\mathrm{t}=2 \mathrm{~min}$. An air-abrasive mixture directed under pressure onto a rigidly fixed saw blade is capable of microcutting due to the kinetic energy of the abrasive particles. At the same time, it is possible to process hard-to-reach places on the parts, if changed the angle of attack of the abrasive and, thus, ensure high-quality processing of low-hard and complex-shaped machine parts.

For abrasive blasting of linter saw teeth, such angles of attack $\alpha$ of abrasive particles are preferred, which lead to intensive microcutting (wear) of the treated surfaces and, as a consequence, the formation of the greatest height of irregularities for capturing cotton fibers during linting. In this case, it is also important to obtain a surface micro-profile, where the irregularities have a sufficient pitch so that several fibers oriented in the cross-section can be fixed in it. These fibers are very likely to be cut and removed from the seeds during the rotation of the saw blades under conditions of force contact. Thus, the lateral edges of the teeth will be involved in the linting work and thus their activation occurs.

\section{Results and discussion}

Experimental research to study the initial state of the teeth of linter saws were carried out on an EMPYREAN XDR X-ray diffractometer (XDR-6100, $2 \mathrm{~kW}$ type), which has the following main characteristics of an X-ray tube and generator: 1) anode material - $\mathrm{Cu}, \mathrm{Co}$, $\mathrm{Fe}, \mathrm{Cr}$; 2) focus size $-1.0 \times 10 \mathrm{~mm}$; 3) maximum power - $2 \mathrm{~kW}$; 4) maximum voltage - 60 $\mathrm{kV}$ (change step 1V); 5) maximum current - $80 \mathrm{~mA}$ (step of change $1 \mathrm{~mA}$ ).

By the method of X-ray diffraction microscopy, a scanned profile of the tops of a linter saw tooth was obtained (Fig. 2).

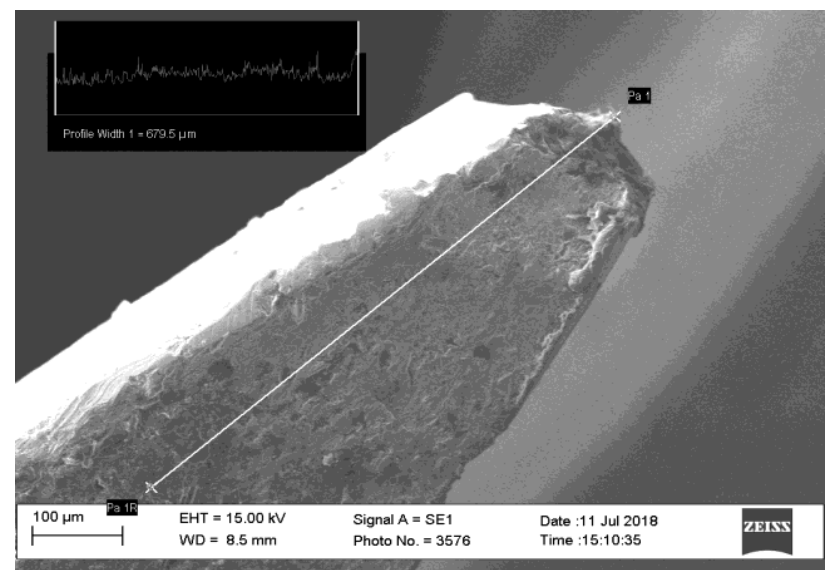

Fig. 2. Scanned microprofile of the tip of the linter saw tooth by X-ray diffraction microscopy

On the front edge of the tooth, burrs are visible that form after the punching operation and that can enhance the linting process in the initial period of work. In the process of tooth wear and smoothing of burrs, their role in the linting process is noticeably reduced. The absence of submicrocracks and the relatively uniform structure of the surface layer indicate the proper quality of the saw blade teeth. 
Fig. 3 shows the scanned areas of the lateral surface of the linter saw tooth. Scanning was carried out in two mutually perpendicular directions (horizontally and vertically) through a selected point at different trajectory lengths (light strip in the images). It was found that the profiles of submicron roughness in two mutually perpendicular directions practically differ little in quantitative and qualitative ratios.

Structural research of the metal surface after abrasive blasting were supplemented by atomic force microscopy [7-10] using an Agilent-5500 scanning probe microscope. A significant advantage of this modern equipment is expressed in the high resolution and accuracy of reproducing the surface micro-profile, as well as in the instant visualization of the studied surface on a computer monitor.

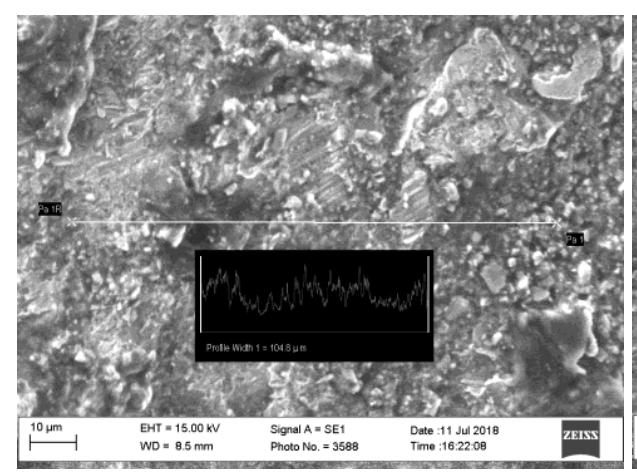

a)

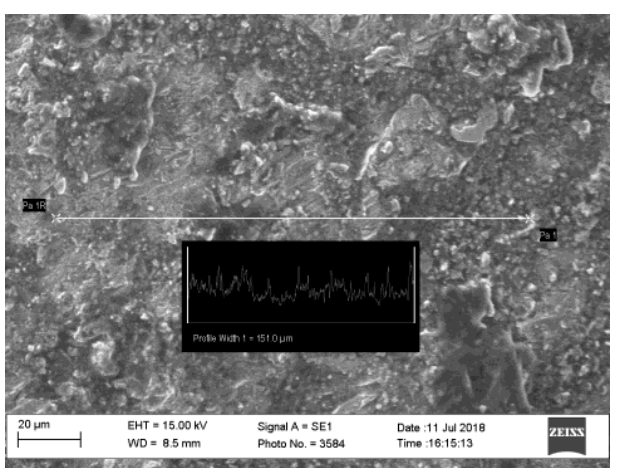

b)

Fig. 3. The scanned micro-profile of the lateral surface of the linter saw tooth near its apex and the profilogram of the surface with submicron irregularities with the horizontal scanning length: $a-104,8$ $\mathrm{mcm} ; \mathrm{b}-151,0 \mathrm{mcm}$

Experimental research of surface irregularities formed after abrasive blasting were carried out on samples cut from a linter saw blade. Analysis of the profilogram of the microprofile of the lateral surface of the teeth without processing, corresponding to a measurement length of $49,8 \mathrm{mcm}$, showed that the maximum height of irregularities and the average pitch of irregularities along the tops are, respectively, $\mathrm{H}=0,143 \mathrm{mcm}$ and $\mathrm{S}=9 \mathrm{mcm}$ (Fig. 4). A similar surface, when $\mathrm{S} / \mathrm{d}<1$ ( $\mathrm{d}$ is the fiber diameter), upon contact with the mass of cotton seeds during linting, is not able to activate and participate in additional cutting of the remaining fibers, since the sizes of the local contact areas are not commensurate with the transverse size of the cotton fibers.

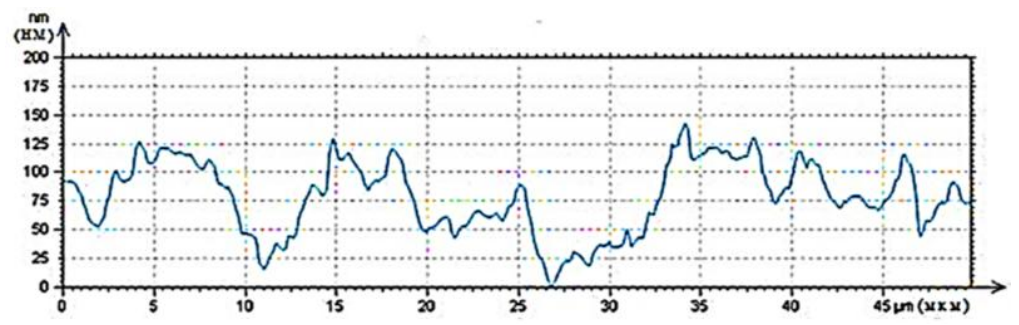

Fig. 4. Scanned profile of the lateral surface of the tooth without processing

Another qualitative and quantitative picture of the scanned profiles of the surfaces of the saw blade teeth was obtained after abrasive blasting (Fig. 5). 


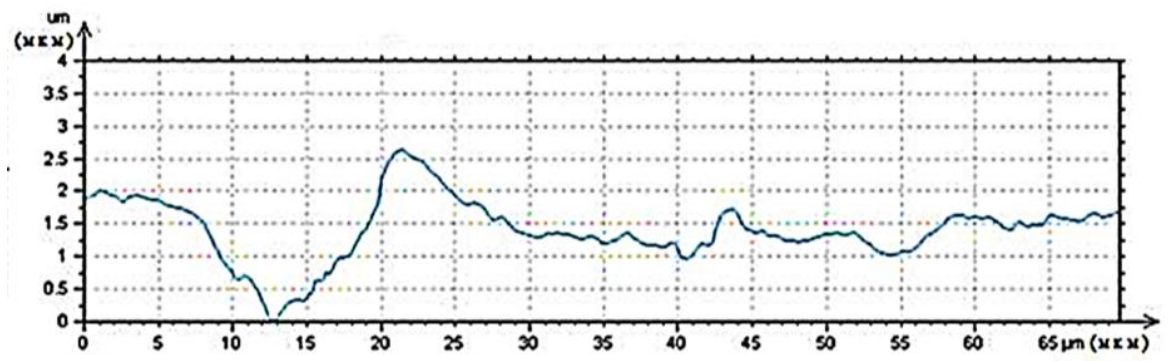

Fig. 5. Scanned profile of the lateral surface of the teeth of linter saws after abrasive blasting

By the method of mathematical planning of the experiment [11] by passing from the coded values of the factors $x_{1}$ and $x_{2}$ to natural values $p$ (air pressure) and $\alpha$ (angle of attack), a regression equation was obtained for the conditional roughness height $H$ :

$$
H=2,3-0,35 p-0,03 \alpha-0,017 p \alpha, \mathrm{mkm}
$$

The adequacy of the linear model, represented by equation (1), was confirmed by testing the hypothesis using Fisher's F-test, and the statistical significance of the coefficients of the regression equation was verified by comparing the absolute values of the coefficients with a confidence interval in accordance with the Student's criterion.

Within the scan lengths of 49,8 and $69,7 \mathrm{mcm}$, there are maximum values of the irregularity heights equal to 2,68 and 2,64 mcm, respectively, i.e. an order of magnitude more than untreated teeth. But the main result of abrasive blasting of teeth of linear saws is the formation of a micro-profile with a significant irregularity pitch exceeding $20 \mathrm{mcm}$, and comparable to the diameter of cotton fibers $(11,3 \ldots 17,7 \mathrm{mcm})$, usually calculated by the equation [12]:

$$
d_{p}=0,0357 \sqrt{\frac{T}{\delta}}, \mathrm{mm}
$$

where, $T$ is the linear density of the fiber, equal to $0,13 \ldots 0,22$ tex $(\mathrm{mg} / \mathrm{m})$ and $\delta$ - average fiber density equal to $0,9 \ldots 1,3 \mathrm{mg} / \mathrm{mm}^{3}$.

Thus, abrasive blasting of the teeth of the linter saw blades creates good prerequisites for enhancing the gripping power of the fibers formed by irregularities and their subsequent shearing.

S shown by visual observations on untreated teeth, the fibers are concentrated mainly on the apex of the tooth, and on processed teeth, a large number of fibers are fixed on the lateral surfaces of the teeth, which confirms the fact that these surfaces are activated by irregularities in the microprofile of the teeth. As established by experiments, the most favorable micro-profile for activating the surface of the teeth during linting corresponds to the processing mode at a pressure of $p=3$ atm and an angle of attack $\alpha=45^{\circ}$.

It is important to note that the height of surface roughness during abrasive blasting is inextricably linked to surface wear caused by the abrasive jet. On the basis of experimental studies of the wear and angle of attack of abrasive strings [13,14], the authors found that there is an extreme dependence between these parameters with an inflection point at an angle of attack $\alpha=45$ and $60^{\circ}$, which is typical for steels and cast irons of different grades, types of heat treatment (normalization and hardening) and hardness range HB 180...575. Taking into account the material of the saw blades (U8G steel, HRA 67...70), the angle of 


\section{ICECAE 2021}

attack $\alpha=45$ of abrasive particles made of black silicon carbide provides an optimal combination of the height and step roughness parameter.

Abrasive blasting of the flank surfaces of the teeth of linter saws consists in microcutting (scratching) that occurs when an abrasive particle strikes at an angle to the surface to be treated. Since microcutting is carried out due to plastic deformation of the contact layers of the metal, the effect of strain hardening (work hardening) should be expected. Strengthening is also facilitated by the impact character of the contact interaction between the abrasive particles and the metal barrier.

To assess the strain hardening, the microhardness was measured on a PMT-3 device according to the standard technique on special specimens-oblique microsections (oblique cut) cut from a saw blade (Fig. 6).
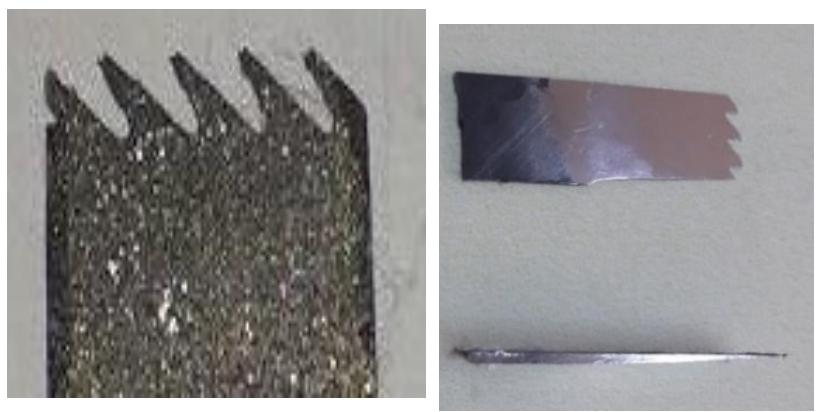

Fig. 6. Special samples - microsections cut from linter saws with preparation of the controlled surface in the form of an oblique cut

The results of an experimental study of the microhardness of the surface layer of U8G steel samples indicate that the depth and degree of hardening increase with an increase in the pressure of the abrasive-air strings (Fig. 7).

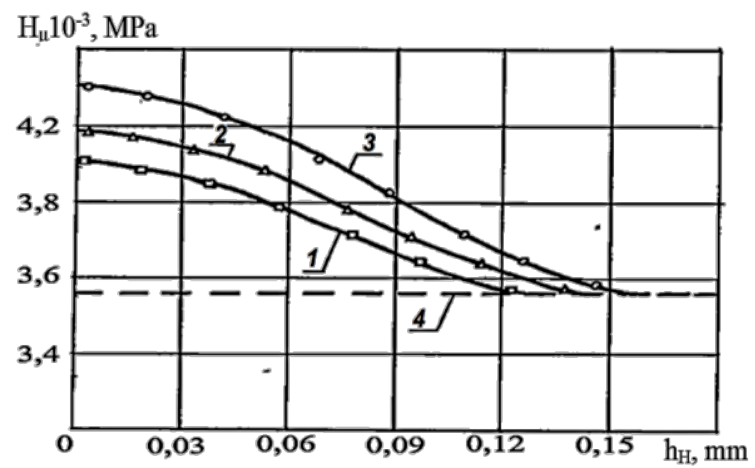

Fig. 7. Change in microhardness $\mathrm{H} \mu$ along the depth of the surface layer hн of U8G steel depending on pressure $p$ during abrasive blasting: $1-p=2$ atm; 2 - $p=3$ atm; $3-p=4$ atm; 4 - H $\mu$ in the initial state, equal to $3560 \mathrm{MPa}$. The angle of attack of the abrasive $\alpha=450$; processing time $\mathrm{t}=2 \mathrm{~min}$

The degree of work hardening $\mathrm{U}$, determined by the equation,

$$
U=\frac{H_{\mu}^{\max }-H_{\mu}^{\text {outg }}}{H_{\mu}^{\text {outg }}} \cdot 100, \%
$$


reaches the highest value at air pressure $p=4$ atm and is $24,2 \%$, and the maximum microhardness $H_{\mu}^{\operatorname{mx}}=4420 \mathrm{MPa}\left(\mathrm{N} / \mathrm{mm}^{2}\right)$ at initial microhardness $H_{\mu}^{\max }=3560 \mathrm{MPa}$. Work hardening depth $h_{H}$ at $p=4$ atm is equal to $0.162 \mathrm{~mm}$.

\section{Conclusions}

Industrial tests and the introduction of abrasive blasting at the cotton ginning industry have shown that the durability of saw blades for linters is doubled or more, which is especially important, since their demand is much higher than that of genie saws. These test results confirm the positive influence of the effect of strain hardening on the wear resistance of saw blades for gins, the teeth of which were subjected to shot blasting with micro beads [4]. The intensification of the linting process due to the activation of the side surfaces of the saw blade teeth is proved by the results of laboratory studies of the quality of seeds: pubescence (fiber preservation) after linting with experimental saws is $23,6 \%$ less than when linting with factory saws.

\section{References}

1. G. Miroshnichenko, Mechanical Engineering 458-468 (1972)

2. E. Zikrieev, Primary processing of raw cotton, 400 (Mekhnat Press, Tashkent, 1999)

3. I. Maksudov, A. Nuraliev, Reference book of the primary processing of cotton, 395 (Mekhnat Press, Tashkent, 1995)

4. I. Shin, A. Juraev, Problems of Textiles 1, 7-11 (2009)

5. I. Shin, Hardening technologies and coatings 10, 24-26 (2010)

6. I. Shin, R. Maksudov, Bulletin of mechanical engineering 4, 44-47 (2011)

7. Z. A. Shodmonkulov, I. Shin, Current problems of innovative technologies of cotton ginning, textile, light industry, printing production and their solutions in the context of science, education, production integration: Abstracts. report Repub. scientific. practical. conf., 138-141 (2019)

8. YU. Nagornov, I. Yasnikov, M. Tyurkov, Methods for surface investigation by atomic force and electron microscopy, 58 (TSU Press, Togliatti, 2012)

9. Z. Shodmonkulov, A. Atakhanov, A. Gulamov, I. Shin, International Journal of Advanced Research in Science, Engineering and Technology 6(8), 11 (2019)

10. A. Spiridonov, Mechanical Engineering 4, 14 (1981)

11. G. Kukin, A. Soloviev, A. Koblyakov, Textile materials science, 352 (Legprombytizdat Press, Moscow, 1989)

12. V. Vinogradov, G. Sorokin, G. Kolokolnikov, Mechanical Engineering 6, 22 (1990)

13. D. Ryakhin, Friction and wear 5(6), 103-108 (1984)

14. G. Hitchings, Wear 70(3), 269-281 (1981) 